Draft Version August 25, 2015

Preprint typeset using $\mathrm{LAT}_{\mathrm{E}} \mathrm{X}$ style emulateapj v. 08/22/09

\title{
PUSHING THE LIMITS, EPISODE 2: K2 OBSERVATIONS OF EXTRAGALACTIC RR LYRAE STARS IN THE DWARF GALAXY LEO IV
}

\author{
L. Molnár ${ }^{1}$, A. PÁl ${ }^{1,2}$, E. Plachy ${ }^{1}$, V. Ripepi ${ }^{3}$, M. I. Moretti ${ }^{4,5}$, R. Szabó ${ }^{1}$, And L. L. Kiss ${ }^{1,6,7}$ \\ Draft version August 25, 2015
}

\section{ABSTRACT}

We present the first observations of extragalactic pulsating stars in the K2 ecliptic survey of the Kepler space telescope. Variability of all three RR Lyrae stars in the dwarf spheroidal galaxy Leo IV were successfully detected, at a brightness of $K p \approx 21.5 \mathrm{mag}$, from data collected during Campaign 1. We identified one modulated star and another likely Blazhko candidate with periods of $29.8 \pm 0.9 \mathrm{~d}$ and more than $80 \mathrm{~d}$, respectively. EPIC 210282473 represents the first star beyond the Magellanic Clouds for which the Blazhko period and cycle-to-cycle variations in the modulation were unambiguously measured.The photometric $[\mathrm{Fe} / \mathrm{H}]$ indices of the stars agree with earlier results that Leo IV is a very metal-poor galaxy. Two out of three stars blend with brighter background galaxies in the K2 frames. We demonstrate that image subtraction can be reliably used to extract photometry from faint confused sources that will be crucial not only for the K2 mission but for future space photometric missions as well.

Subject headings: stars: variables: RR Lyrae — methods: observational — techniques: photometric

\section{INTRODUCTION}

Kepler has provided incredible results on extrasolar planets and planetary systems, as well as on stellar astrophysics. The space telescope was designed to be the most precise photometer ever built in order to detect the transits of numerous small planets (Borucki et al. 2010). To achieve this goal, the original mission of $\mathrm{Ke}$ pler focused almost exclusively on the Galactic stellar population, approximately 170000 targets, within the Lyra-Cygnus field. But after the failure of two reaction wheels the telescope permanently lost its ability to maintain its original attitude. Soon a new mission, called K2, was initiated to save the otherwise healthy and capable space telescope (Howell et al. 2014). Since then, Kepler has been observing in shorter, 75 day long campaigns along the Ecliptic to balance the radiation pressure from the Sun.

The new fields opened the possibility to extend the capabilities of Kepler into new areas and we set out to explore the limits of the K2 mission. Molnár et al. (2015) investigated the first observations of field RR Lyrae stars. Szabó et al. (2015) and Pál et al. (2015) showed that Kepler can be used for Solar System research, including the detection of main-belt asteroids and trans-neptunian objects (TNOs). In this paper we continue this work, ex-

\footnotetext{
Electronic address: molnar.laszlo@csfk.mta.hu

${ }^{1}$ Konkoly Observatory, Research Centre for Astronomy and Earth Sciences, Hungarian Academy of Sciences, H-1121 Budapest, Konkoly Thege Miklós út 15-17, Hungary

${ }^{2}$ Eötvös Loránd Tudományegyetem, H-1117 Pázmány Péter sétány 1/A, Budapest, Hungary

${ }^{3}$ INAF - Osservatorio Astronomico di Capodimonte, Via Moiariello 16, I-80131 Naples, Italy

${ }^{4}$ INAF - Osservatorio Astronomico di Bologna, via Ranzani 1, 40127 Bologna, Italy

${ }_{5}^{5}$ Scuola Normale Superiore di Pisa, piazza dei Cavalieri 7, 56126 Pisa, Italy

${ }^{6}$ Gothard-Lendület Research Team, H-9704 Szombathely, Szent Imre herceg út 112, Hungary

${ }^{7}$ Sydney Institute for Astronomy, School of Physics A28, University of Sydney, NSW 2006, Australia
}

panding the reach of Kepler towards extragalactic pulsating stars. The three RR Lyrae stars in the galaxy Leo IV are the first non-cataclysmic stellar targets $\mathrm{Ke}$ pler ever detected outside the Galaxy. We note in passing that the space telescope has already observed four supernovae during the original mission (Olling et al. 2015).

Leo IV is one of the small and faint dwarf spheroidal galaxies around the Milky Way that were recently discovered with the help of the Sloan Digital Sky Survey, with a mass and luminosity of $M=(1.4 \pm 1.5) \cdot 10^{6} \mathrm{M}_{\odot}$ and $M_{V}=-5.1 \pm 0.6 \mathrm{mag}$ (Belokurov et al. 2007: Simon \& Geha 2007). It is located at a heliocentric distance of $154 \pm 5 \mathrm{kpc}$, and has a half-light radius of $3.3 \mathrm{arcmin}$ translating to a physical size of $160 \mathrm{pc}$ (Moretti et al. 2009). Most of the stars in Leo IV are older than 12 Gyr, although another, brief episode of star formation likely occurred 1-2 Gyr ago (Sand et al. 2010). The galaxy is very metal-poor: different studies determined the average metallicity to be around $\langle[\mathrm{Fe} / H]\rangle=-2.3$ (Simon \& Geha 2007: Sand et al. 2010) or possibly as low as $\left\langle\left[F^{\prime} e / H\right]\right\rangle=-2.58$ (Kirby et al. 2008). The galaxy was also surveyed by Moretti et al. (2009) who discovered four variable stars, three fundamental-mode RR Lyrae (RRab) and a single SX Phe pulsator.

RR Lyrae stars are ubiquitous in all nearby galaxies and have been detected even beyond the Local Group (Da Costa et al. 2010), but our knowledge decreases with distance. Our closest neighbors, the Magellanic Clouds and the Sagittarius dwarf and its stellar stream have been surveyed extensively both in terms of stars and temporal coverage, thanks to the MACHO and OGLE programs (see, for example, Alcock et al. 1996 Soszynski et al. 2009, 2010, 2014). But studies of other galaxies, especially of those beyond $80-100 \mathrm{kpc}$, are based on less amounts of data. These observations are usually aimed at distance determination and population studies.

Extragalactic surveys have their limitations, however. Multiple studies revealed several candidate Blazhko stars. Stetson et al. (2014) for example, identified 24 stars with signs of amplitude variation out of 194 


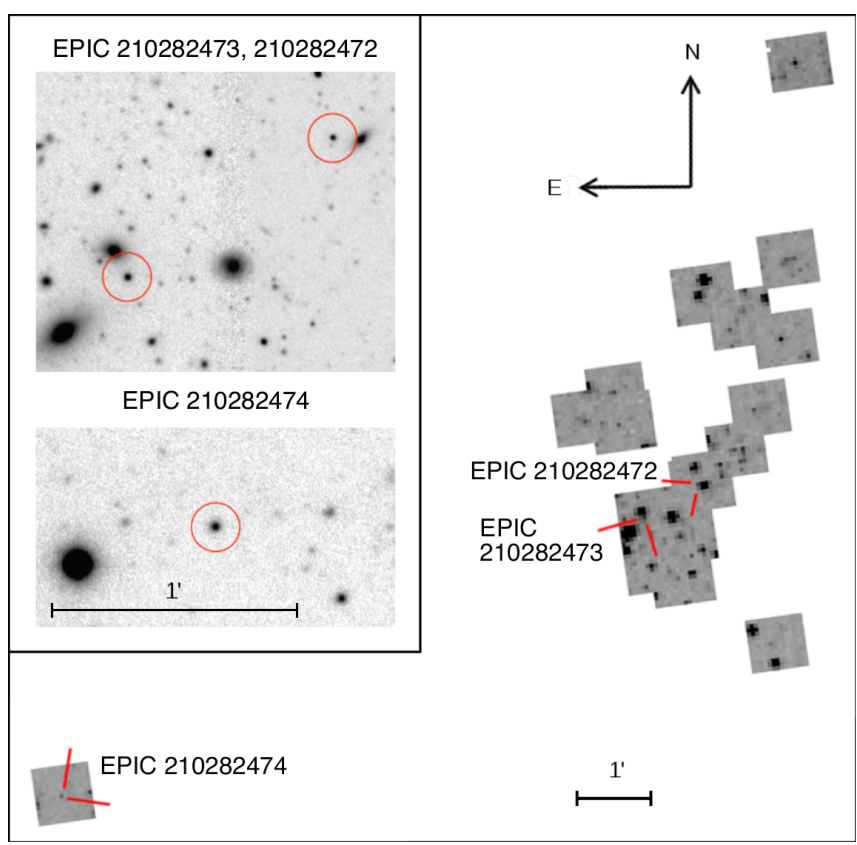

FIG. 1. - The K2 target pixel masks covering parts of Leo IV. The three RR Lyrae stars are indicated. The insert shows highresolution deep imagery obtained by combining data from the William Herschel Telescope, the Isaac Newton Telescope, and the Southern Astrophysical Research telescope, collected by Moretti et al. (2009). Red circles represent the apertures used in the reduction of the $\mathrm{K} 2$ data (see Figure 2).

RR Lyrae stars in Leo I, and we know one candidate in M31 too (Brown et al. 2007). These low occurrences are likely just lower limits: we now know that about $50 \%$ of RRab stars are modulated in the Milky Way (Jurcsik et al. 2009. Benkö et al. 2014). But in order to determine the accurate occurrence rates and modulation periods of Blazhko stars, one usually requires several weeks of intensive observations, and thus unambiguous detections have not yet been made. Also, data from Kepler revealed that continuous observations are the key to detect any cycle-to-cycle variations - another feature that earlier studies lacked (Szabó et al. 2010). Therefore we set out to gather the first extended, uninterrupted observations from extragalactic pulsating stars with the help of $\mathrm{K} 2$.

\section{OBSERVATIONS AND DATA REDUCTION}

Leo IV fell into the field-of-view of the first scientific campaign ( $\mathrm{C} 1$ ) of the $\mathrm{K} 2$ mission and was observed between May 30 and August 21, 2014, corresponding to Barycentric Julian Day (BJD) 2456808.18-2456890.33. We requested to observe the $3 \mathrm{RR}$ Lyrae variables and the 16 brightest giant stars in the galaxy through the K2 Guest Observer proposal GO1019 in long cadence mode, with an integration time of $29.4 \mathrm{~min}$. We estimated the maximum brightness of the RR Lyrae stars to be around $K p=21-21.2 \mathrm{mag}$ in the Kepler passband, leading to a precision of a few tenths of a magnitude per long cadence data point. At that time the much fainter SX Phe star $(V=23.0 \mathrm{mag})$ seemed to be beyond the capabilities of Kepler so we did not include it in the proposal, although the recent experiences with the similarly faint TNO, $2002 \mathrm{GV}_{31}$, suggest that we could have detected its variation too (Pál et al. 2015). In this paper we focus on
TABLE 1

Summary of the K2 OBSERVATIONS OF THE RR Ly TARGETS IN THE DWARF GALAXY LEO IV.

\begin{tabular}{lccccc}
\hline \hline Object & $\begin{array}{c}\text { Mask } \\
(\mathrm{px})\end{array}$ & $\begin{array}{c}\text { R.A. } \\
(\mathrm{J} 2000)\end{array}$ & $\begin{array}{c}\text { Decl. } \\
(\mathrm{J} 2000)\end{array}$ & $\begin{array}{c}V \\
(\mathrm{mag})\end{array}$ & ID \\
\hline 210282472 & $15 \times 14$ & $11^{\mathrm{h}} 32^{\mathrm{m}} 55.8^{\mathrm{s}}$ & $-0^{\circ} 33^{\prime} 29.4^{\prime \prime}$ & 21.46 & V2 \\
210282473 & $15 \times 13$ & $11^{\mathrm{h}} 32^{\mathrm{m}} 59.2^{\mathrm{s}}$ & $-0^{\circ} 34^{\prime} 03.6^{\prime \prime}$ & 21.47 & V1 \\
210282474 & $14 \times 14$ & $11^{\mathrm{h}} 33^{\mathrm{m}} 36.6^{\mathrm{s}}$ & $-0^{\circ} 38^{\prime} 43.3^{\prime \prime}$ & 21.52 & V3 \\
\hline Note. - IDs refer to the identifications given by & Moretti et al. \\
2009 . & & & &
\end{tabular}

the three RR Lyrae stars from the sample. The summary of their observation parameters is shown in Table 1.

For all of the tasks described below we employed exclusively the utilities shipped within the FiTsH ${ }^{8}$ (Pál 2012). FITSH is a lightweight, yet comprehensive, fully open source astronomical data reduction and analysis software package. It is comprised of a collection of standalone binary programs that are utilized through various UNIX shell scripts. The accurate photometric time series for these three extragalactic Leo IV RR Lyr targets have been obtained as follows. The image scale of Kepler is $3.98^{\prime \prime} / \mathrm{px}$, and the point spread functions are at least 3 5 px wide that leads to confusion of nearby sources. Although Leo IV is sparse enough to be resolved even with Kepler, two out of the three RR Lyr targets were extremely close to bright background galaxies (i.e., nearly within a pixel, see Figures 1 and 2), accurate photometry can only be done by involving image subtraction techniques. Since the pointing jitter of Kepler in the K2 mission was in the range of a pixel, the individual frames had to be adjusted to the same reference system before performing any kind of differential image analysis. In order to precisely estimate the shifts and rotations between the (subsequent) frames, we involved additional K2 frames since the raw K2 data do not include a frame-by-frame centroid coordinate. These additional 14 fields were the target pixel files of EPIC 201424914, 201430029 and the 12 stamps having an identifier between 210282475 and 210282486, all from the GO1019 proposal (where EPIC refers to the K2 Ecliptic Plane Input Catalog). These $3+14$ stamps were combined to a single image and the foreground stars were used to obtain precisely the transformation between these images (Figure 1).

Since the K2 mission observes along the ecliptic plane in an approximately $10^{\circ}$-wide area, the background of these observations also varies gradually due to the increasing amount of zodiacal light throughout the campaign. Hence, prior any differential analysis, the background must also be subtracted. Luckily, the fields containing the 3 RR Lyr targets as well as the additional 14 stamps were not crowded, and the background can simply be determined by considering the median of all observed pixel values.

After the frames were registered to the same reference system and the background was subtracted accordingly, we chose every 20th image to obtain median-averaged master frame used as a reference for image subtraction. Since the number of stars having a good signal-to-noise ratio in these frames were insufficient and the instrument

\footnotetext{
8 http://fitsh.szofi.net/
} 

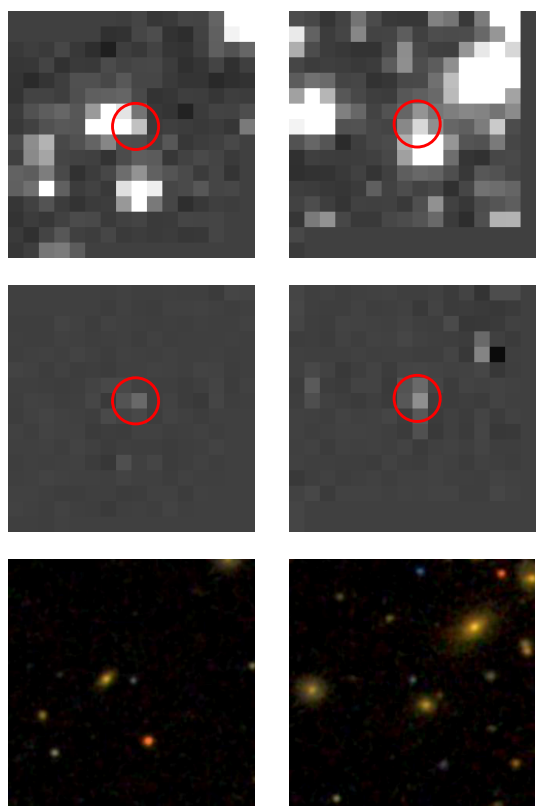

FIG. 2.- Image stamps showing the vicinity of the K2 extragalactic RR Lyrae targets 210282472, 210282473 and 210282474. The first row shows the stamps on the master median-combined image used in the process of differential photometry. The second row shows stamps created as the average of a roughly dozen differential images taken at the brightest phase of the RR Lyr oscillations. The photometric aperture with an $r=1.5 \mathrm{px}$ radius used in the procedure is shown as a red circle. Stamps in the third row show the respective SDSS DR9 images. All of these stamps cover an area of $64^{\prime \prime} \times 64^{\prime \prime}$ on the sky, equivalent to $16 \times 16 \mathrm{Kepler}$ pixels.

does not show any significant variation in its point-spread function, we did not perform any cross-convolution between the adjusted images. This procedure also simplified the evaluation of the photometry and hence it was not necessary to perform photometry on convolved apertures (Pál 2009). Therefore, the final fluxes were obtained as the sum of the flux in the subtracted frame and in the master frame, i.e., $F_{i}=F_{i, s u b t r}+F_{\text {master }}$. For these flux estimations we used simple aperture photometry with an aperture radius of $r=1.5 \mathrm{px}$ (Figure 2). However, we must note that the reference flux on the master frame can only be estimated with an additional systematic uncertainty in the case of the confused sources (210282472 and 210282473). Namely, we compared the peak pixel values on the stellar sources with the fluxes obtained using an aperture of $r=1.5 \mathrm{px}$. The ratio of these two numbers aid the estimation of the reference flux in the case of the confused sources as well and we found that the systematic uncertainty of this method is in the range of $2-3 \%$ for these sources. Due to the nature of the differential photometry, this kind of systematics yields a systematic amplification in the variability amplitudes. The random errors in the photometric fluxes have been estimated using the photon noise derived from the instrumental gain of $113.3 \mathrm{e}^{-} / \mathrm{ADU}$ as well as the standard deviation of the background pixels near the target sources. Due to the differential processing, this latter component also includes the photon noise of the nearby confusing sources.
TABLE 2

K2 PHotometric DATA of The RR LyR StARs in LeO IV.

\begin{tabular}{lcccc}
\hline \hline Object & $\begin{array}{c}\text { Time } \\
\text { (BJD) }\end{array}$ & $\begin{array}{c}\text { Brightness } \\
(R \text { mag })^{\mathrm{a}}\end{array}$ & $\begin{array}{c}\text { Error } \\
(\mathrm{mag})\end{array}$ & Type \\
\hline 210282472 & 2456810.28292 & 20.865 & 0.062 & raw \\
210282472 & 2456810.30336 & 20.659 & 0.048 & raw \\
210282472 & 2456810.32379 & 20.974 & 0.058 & raw \\
$\ldots$ & & & & \\
\hline 210282473 & 2456810.28292 & 21.247 & 0.069 & raw \\
210282473 & 2456810.30336 & 21.188 & 0.069 & raw \\
210282473 & 2456810.32379 & 21.240 & 0.076 & raw \\
$\ldots$ & & & & \\
\hline 210282474 & 2456810.28292 & 21.821 & 0.122 & raw \\
210282474 & 2456810.30336 & 21.585 & 0.093 & raw \\
210282474 & 2456810.32379 & 21.565 & 0.085 & raw \\
$\ldots$ & & & & \\
\hline 210282472 & 2456810.28292 & 20.886 & 0.062 & proc \\
210282472 & 2456810.32379 & 20.995 & 0.058 & proc \\
210282472 & 2456810.34422 & 21.004 & 0.054 & proc \\
$\ldots$ & & & &
\end{tabular}

Note. - Table 2 is published in its entirety in the electronic edition of the Astrophysical Journal Letters. A portion is shown here for guidance regarding its form and content.

a Magnitudes shown here are transformed to USNO-B1.0 $R$ system, see text for further details. Indices "raw" and "proc" correspond to raw and processed (sigma-clipped and Fourierfiltered) data, respectively.

\section{LIGHT CURVE ANALYSIS}

Once the raw light curves were obtained, we removed the outliers from the light curves with a sigma-clipping algorithm. We subtracted an initial Fourier fit from the light curves, clipped the residual at the $3 \sigma$ levels three consecutive times and then restored the original signal.

Finally, we applied Fourier filtering to remove the slow variations. The photometry of these faint targets is very sensitive to the variations in the background flux level. Even the best raw light curves we extracted contained additional variations up to $0.1-0.2 \mathrm{mag}$ in the last $10-20 \mathrm{~d}$ of data. However, a simple high-pass frequency filter would remove any modulation signal along with the low-frequency noise. So instead we removed all lowfrequency components below $0.5 \mathrm{~d}^{-1}$ that were stronger than $10 \mathrm{mmag}$ and were not connected to any potential modulation frequencies. The final light curves are displayed in the left panels of Figure 3. The right panels show the folded light curves, along with the binned phase curves.

The increasing background from the zodiacal light lowered the photometric accuracy of individual data points towards the end of the campaign. The accuracy of a single LC point was similar for EPIC 210282472 and 210282473: 0.050-0.055 mag at the beginning and 0.078$0.092 \mathrm{mag}$ during the last days of the measurements. The errors are somewhat higher for the unblended star EPIC 210282474, starting from $0.087 \mathrm{mag}$ and reaching $0.107 \mathrm{mag}$ at the end of the campaign. 210282472 and 210282473 also appear to be brighter than 210282474 by 0.50 and $0.34 \mathrm{mag}$, respectively. As mentioned, the main component of this difference comes from the uncertainty of the zero-point determination for the confused sources. Similar differences appear in the peakto-peak amplitudes that are lowered to 0.4 and $0.6 \mathrm{mag}$ for 210282472 and 210282473 , compared to 0.94 mag for 210282474. In contrast, Moretti et al. (2009) found the amplitudes to be quite similar: $0.64,0.73$, and 0.65 mag 

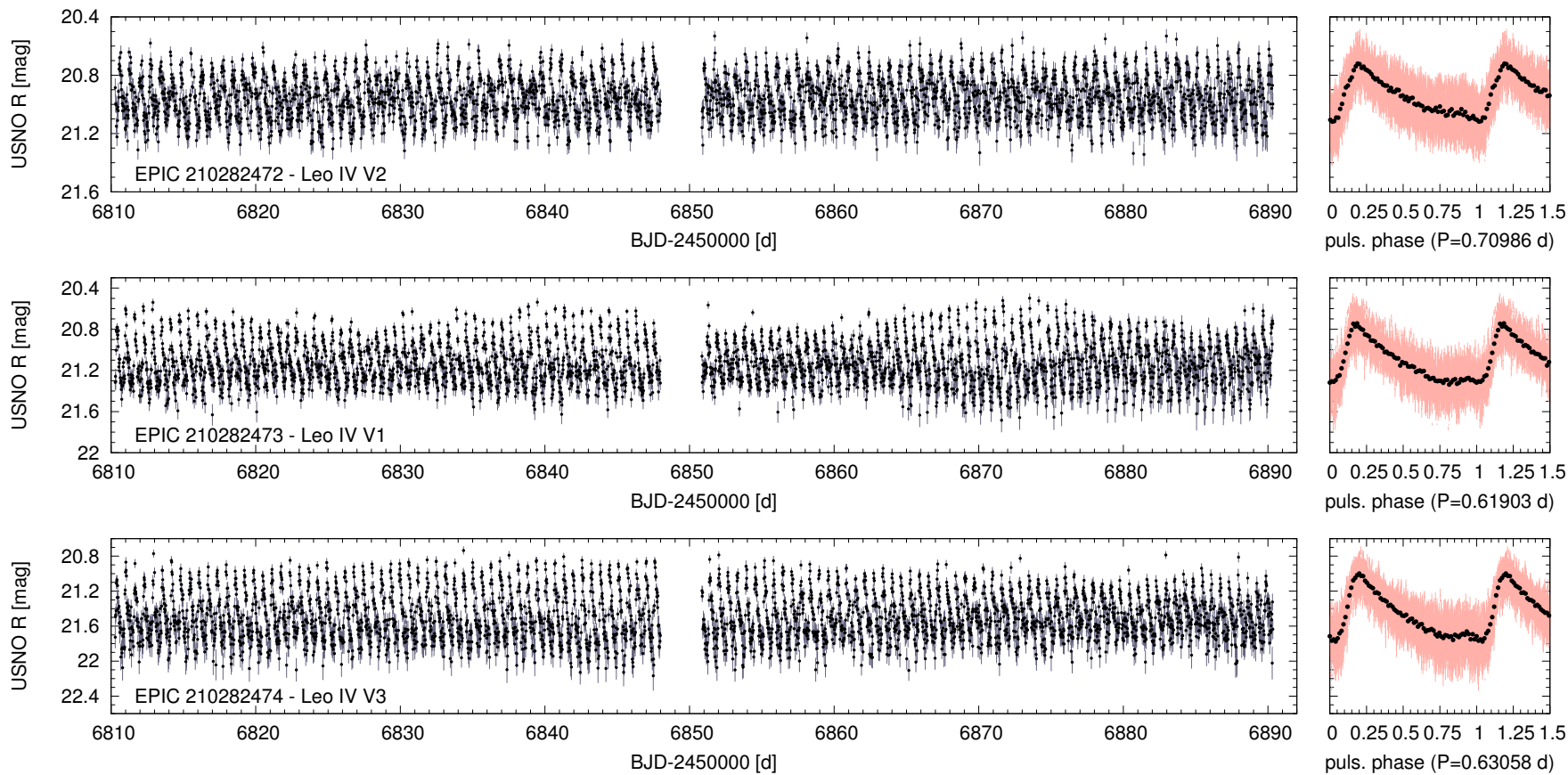

Fig. 3. - Light curves of the three RR Lyrae stars. Left panels: light curves after outlier removal and Fourier filtering. Note the variable amplitude of EPIC 210282473 in the middle panel. Designations from Moretti et al. (2009) are also indicated. The right panels show the folded light curves (pink dots and lines) and the binned phase curves (black points). We used 75 bins per pulsation period for each star.

in $V$ band, respectively. All these differences between EPIC 210282474 and the other two stars indicate that the confusing sources contribute considerably to the measured flux levels of EPIC 210282472 and 210282473 increasing their brightnesses and lowering the amplitudes and photon noise levels we measure. Nevertheless, this zero-point uncertainty only changes the overall amplitudes but does not distort the shape of the light curves, therefore has no further effect on the astrophysical content of the data.

For all of these three objects, photometric magnitudes have been transformed into USNO-B1.0 $R$ system (Monet et al. 2003). This procedure has been performed similarly as it was done by Pál et al. (2015). The raw and processed photometric data series are displayed in Table 2. Note that the photometric errors shown in this Table do not include the aforementioned zero-point uncertainty.

We carried out a standard Fourier analysis with the PERIOD04 software (Lenz \& Breger 2005), using multifrequency least-squares fits and consecutive prewhitenings. The identified frequency components are listed in Table 3. We detected modulation triplets $\left(n f_{1}\right.$ harmonics and corresponding $n f_{1} \pm f_{m}$ sidepeaks, where $n=1,2,3 \ldots$ ) in the Fourier spectra of EPIC 210282473 and 210282474. We note, however, that in the latter case the length of the data is insufficient to properly resolve the side-peaks, and their distances correspond to the length of the data instead of the modulation period, so we did not include them in the fit.

We could also identify the characteristic signal of the spacecraft attitude correction maneuvers, at $f_{\text {corr }}=$ $4.08 \mathrm{~d}^{-1}$ and its harmonics, but with signal-to-noise ratios below 4. Apparently, very faint objects suffer less from the attitude changes of $\mathrm{K} 2$ than bright targets.
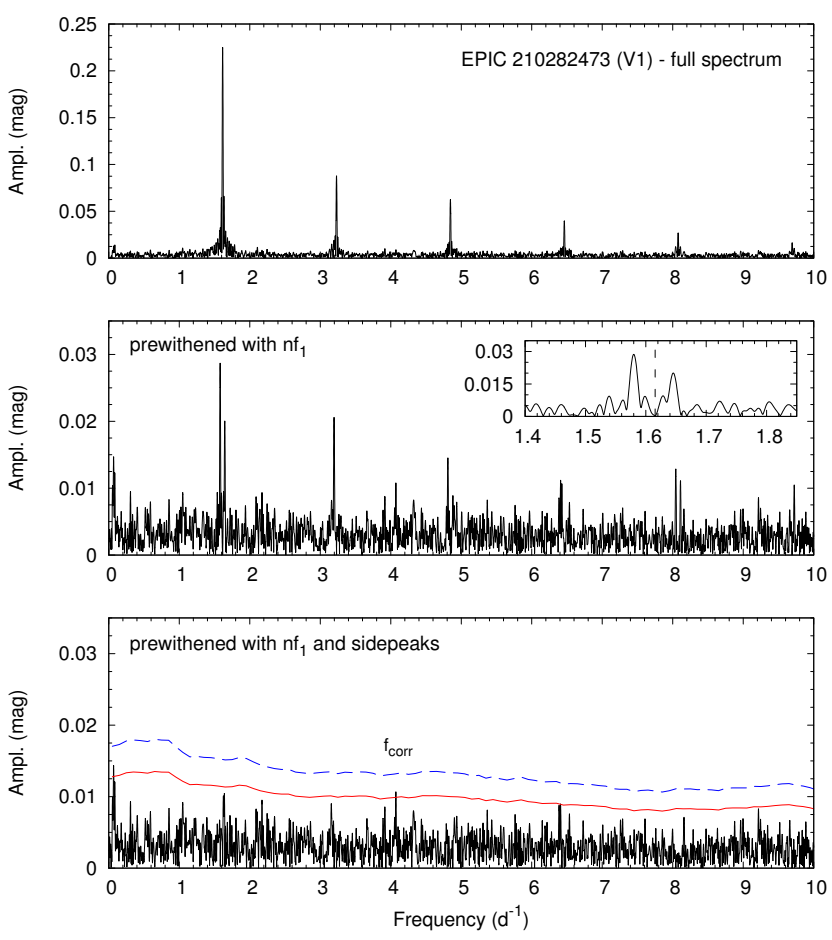

FIG. 4.- Fourier transform of the light curve of EPIC 210282473. The top panel shows the original spectrum. Significant modulation sidepeaks are visible in the middle panel after prewhitening with the main peak and its harmonics. The insert shows the triplet around the position of the main peak (dashed line). The bottom panel shows the residual after the side peaks have been prewhitened as well. The red solid and blue dashed lines are the 3 and 4 SNR levels, respectively. The $f_{\text {corr }}$ label marks the position of the marginally detected spacecraft attitude correction frequency. 

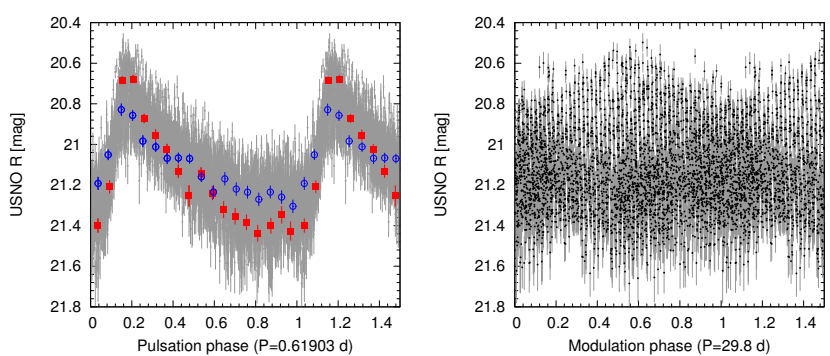

FIG. 5.- Blazhko effect in EPIC 210282473. Left: data folded with the pulsation period. Binned data from 2-day long sections are overlaid, red dots show the maximum-amplitude modulation phase, blue circles show the minimum-amplitude phase. Right: light curve folded with the modulation period.

Other significant frequency peaks, such as low-amplitude additional modes or half-integer peaks related to period doubling - both observed in many modulated RRab stars - did not appear after prewhitening. This is not surprising, however, considering that the 3 SNR (signalto-noise ratio) significance limit in the Fourier spectra is around 8-12 mmag, well above the strongest additional peaks detected in the original Kepler sample. The additional modes described by Benkő et al. (2014) reached only $3 \mathrm{mmag}$, so similar modes would be undetectable in the Leo IV stars. The Fourier spectra of EPIC 210282473 are shown in Figure 4

Such faint targets are very sensitive to any instrumental problems arising both from the observations or the data reduction. Counterintuitively, the light curve of the only unblended star, EPIC 210282474 was the most problematic in our case. Even after the Fourier filtering, some slow, low-amplitude ( $0.1 \mathrm{mag})$ variations still persisted in the last 15 days of the light curve (Figure 3). Examination of the $\mathrm{K} 2$ full-frame images did not reveal any obvious contaminating sources in the same or adjacent modules. The SDSS images did not reveal any close-by objects either (see Figure 2). We visually inspected the stacked image obtained using the observations taken with the $2.5 \mathrm{~m}$ Isaac Newton Telescope, the $4.2 \mathrm{~m}$ William Herschel Telescope, and the $4.1 \mathrm{~m}$ Southern Astrophysical Research Telescope (SOAR) in 2007 by Moretti et al. (2009), but found no significant nearby objects to within $1.0^{\prime \prime}$. This image is displayed in Figure 1 . Images of the other two targets indicate that the confusion is caused almost exclusively by a single bright galaxy near each star. Another star can be detected 2.1" from 210282472, but it is about 3.5 mag fainter than the RR Lyrae target so its contribution is negligible.

\section{RESULTS}

\subsection{Blazhko effect in Leo IV}

One star, EPIC 210282473, is clearly modulated. Although the signs of amplitude changes in RR Lyrae stars have been observed as far as the Andromeda galaxy (Brown et al. 2007), this is the first star beyond the Magellanic Clouds for which the detailed modulation properties can be determined. The K2 data covers almost three Blazhko cycles. The distance of the triplet side peaks $\left(n f_{1} \pm f_{m}\right)$ suggested a modulation period of $P_{m}=30 \pm 3 \mathrm{~d}$, but the independent fits to the modulation peaks resulted in large uncertainties. We also calculated the amplitude and phase variations $\left(A_{1}, \phi_{1}\right)$ of the main frequency peak $\left(f_{1}\right)$ by dividing the data into short segments. A sine fit to $A_{1}(t)$ itself led to a more precise Blazhko period: $P_{m}=29.8 \pm 0.9 \mathrm{~d}$.

Figure 5 shows the modulation properties of EPIC 210282473. The right panel is the light curve, folded with the modulation period: the presence of the Blazhko effect is even more clear here than in the normal light curve. The left panel illustrates how the shape of the light curve itself changes over a modulation cycle. We selected two 2-day long sections of a low- and a high-amplitude state, respectively, and calculated the phase-binned values of the data. Red squares and blue circles show the two states, respectively: the differences in amplitude are evident around maximum and minimum light, but the phases at which the extrema occur show little to no shifts.

Another representation is included in Figure 6 where the upper and middle panels show the variation of the $A_{1}$ and $\phi_{1}$ Fourier-terms. These figures confirm that there is significant amplitude variation in the data, but the phase modulation is almost negligible in the star. Moreover, the middle column of Figure 6 indicates that the cycles are not repetitive and the amplitude and phase variation are not strictly correlated to each other, similar to some modulated stars in the original Kepler sample (Benkö et al. 2014).

The case of EPIC 210282474 is more ambiguous, since the light curve of the star suffers from instrumental effects more than those of the other two. The Fourierfiltered light curve shows some amplitude and phase variation but those are suspiciously - but not exactlysymmetric to the mid-campaign data download period. This symmetry in the amplitudes invites to suspect some kind of instrumental origin, although we could not identify any contaminating source. We also detect weak phase variations that seem to follow the changes in pulsation amplitude (right column of Figure 6). If we accept that these variations indeed originate from modulation, its period is clearly longer than the length of the campaign. We identified side-peaks in the frequency spectrum, but given the limited length of the data, they are not resolved properly. Overall, since we detect the variations in the first, better-quality part of the light curve as well, we accept this signal as a likely (although not unquestionable) detection of the Blazhko effect. Most stars in the Milky Way have modulation periods longer than 10-20 d (Szczygieł \& Fabrycky 2007): the values we determined for the Leo IV stars agree with that period distribution. Although the Leo IV sample is very limited, it confirms that the Blazhko effect is abundant in other galaxies as well.

EPIC 210282472 also shows some phase variations but only marginal changes in amplitude (left column of Figure 6). However, these amplitude changes can be partly attributed to the large scatter of the light curve points that make the phase determination uncertain for shorter data segments (10 days per segment in this case). Given the lack of significant amplitude variations, we classify this star as non-modulated.

We also calculated the Fourier phase values for the original observations of Moretti et al. (2009) with the frequencies determined from the $\mathrm{K} 2$ data, and transformed them to the more classical $\mathrm{O}-\mathrm{C}$ diagrams. The results, shown in the bottom row of Figure 6, suggest that pul- 
TABLE 3

FREQUENCY TABLES FOR EPIC 210282472, 210282473 AND 210282474.

\begin{tabular}{|c|c|c|c|c|c|c|c|}
\hline EPIC & Freq. ID & Freq $\left(d^{-1}\right)$ & Amp (mag) & $\phi(\operatorname{rad} / 2 \pi)$ & $\pm \mathrm{F}\left(\mathrm{d}^{-1}\right)$ & $\pm \mathrm{A}$ (mag) & $\pm \phi(\operatorname{rad} / 2 \pi)$ \\
\hline $\begin{array}{l}210282472 \\
- \\
- \\
- \\
- \\
\end{array}$ & $\begin{array}{l}f_{0} \\
2 f_{0} \\
3 f_{0} \\
4 f_{0} \\
5 f_{0} \\
\end{array}$ & $\begin{array}{c}1.40872 \\
2.81745 \\
4.22617 \\
5.6349 \\
7.0436 \\
\end{array}$ & $\begin{array}{l}0.1400 \\
0.0640 \\
0.0427 \\
0.0217 \\
0.0106 \\
\end{array}$ & $\begin{array}{c}0.1786 \\
0.7512 \\
0.380 \\
0.999 \\
0.679 \\
\end{array}$ & $\begin{array}{c}0.00011 \\
0.00023 \\
0.00035 \\
0.0007 \\
0.0014 \\
\end{array}$ & $\begin{array}{l}0.0023 \\
0.0023 \\
0.0023 \\
0.0020 \\
0.0019 \\
\end{array}$ & $\begin{array}{c}0.0024 \\
0.0053 \\
0.008 \\
0.016 \\
0.032 \\
\end{array}$ \\
\hline $\begin{array}{l}210282473 \\
- \\
- \\
- \\
- \\
- \\
- \\
- \\
- \\
- \\
- \\
- \\
- \\
- \\
\end{array}$ & $\begin{array}{l}f_{0} \\
2 f_{0} \\
3 f_{0} \\
4 f_{0} \\
5 f_{0} \\
6 f_{0} \\
f_{0}-f_{m} \\
f_{0}+f_{m} \\
2 f_{0}-f_{m} \\
3 f_{0}-f_{m} \\
4 f_{0}-f_{m} \\
5 f_{0}-f_{m} \\
5 f_{0}+f_{m} \\
6 f_{0}+f_{m} \\
\end{array}$ & $\begin{array}{c}1.61545 \\
3.23090 \\
4.84635 \\
6.46181 \\
8.0773 \\
9.6927 \\
1.5818 \\
1.6491 \\
3.1973 \\
4.8127 \\
6.4282 \\
8.0436 \\
8.1109 \\
9.7263 \\
\end{array}$ & $\begin{array}{l}0.2249 \\
0.0869 \\
0.0616 \\
0.0405 \\
0.0265 \\
0.0152 \\
0.0261 \\
0.0150 \\
0.0199 \\
0.0132 \\
0.0104 \\
0.0119 \\
0.0098 \\
0.0095 \\
\end{array}$ & $\begin{array}{c}0.1459 \\
0.6798 \\
0.2126 \\
0.801 \\
0.349 \\
0.950 \\
0.016 \\
0.344 \\
0.485 \\
0.998 \\
0.507 \\
0.144 \\
0.436 \\
0.097 \\
\end{array}$ & $\begin{array}{c}0.00008 \\
0.00020 \\
0.00028 \\
0.00042 \\
0.0006 \\
0.0011 \\
0.0006 \\
0.0012 \\
0.0008 \\
0.0012 \\
0.0016 \\
0.0014 \\
0.0018 \\
0.0018 \\
\end{array}$ & $\begin{array}{l}0.0032 \\
0.0032 \\
0.0031 \\
0.0031 \\
0.0030 \\
0.0028 \\
0.0025 \\
0.0031 \\
0.0030 \\
0.0030 \\
0.0031 \\
0.0031 \\
0.0029 \\
0.0029 \\
\end{array}$ & $\begin{array}{c}0.0017 \\
0.0045 \\
0.0064 \\
0.010 \\
0.015 \\
0.026 \\
0.015 \\
0.029 \\
0.019 \\
0.028 \\
0.037 \\
0.033 \\
0.041 \\
0.043 \\
\end{array}$ \\
\hline $\begin{array}{l}210282474 \\
- \\
- \\
- \\
- \\
-\end{array}$ & $\begin{array}{l}f_{0} \\
2 f_{0} \\
3 f_{0} \\
4 f_{0} \\
5 f_{0} \\
6 f_{0}\end{array}$ & $\begin{array}{c}1.58582 \\
3.17164 \\
4.75747 \\
6.34329 \\
7.9291 \\
9.5149\end{array}$ & $\begin{array}{l}0.2899 \\
0.1219 \\
0.0846 \\
0.0553 \\
0.0313 \\
0.0136\end{array}$ & $\begin{array}{c}0.7674 \\
0.9159 \\
0.0845 \\
0.300 \\
0.516 \\
0.677\end{array}$ & $\begin{array}{c}0.00007 \\
0.00018 \\
0.00025 \\
0.00039 \\
0.0007 \\
0.0016\end{array}$ & $\begin{array}{l}0.0032 \\
0.0032 \\
0.0036 \\
0.0035 \\
0.0035 \\
0.0035\end{array}$ & $\begin{array}{c}0.0017 \\
0.0041 \\
0.0058 \\
0.009 \\
0.016 \\
0.036\end{array}$ \\
\hline
\end{tabular}

Note. - Frequencies, amplitudes, phases and their respective uncertainties. The modulation frequency for EPIC 210282473 was set to $f_{m}=0.03361 \mathrm{~d}^{-1}$.
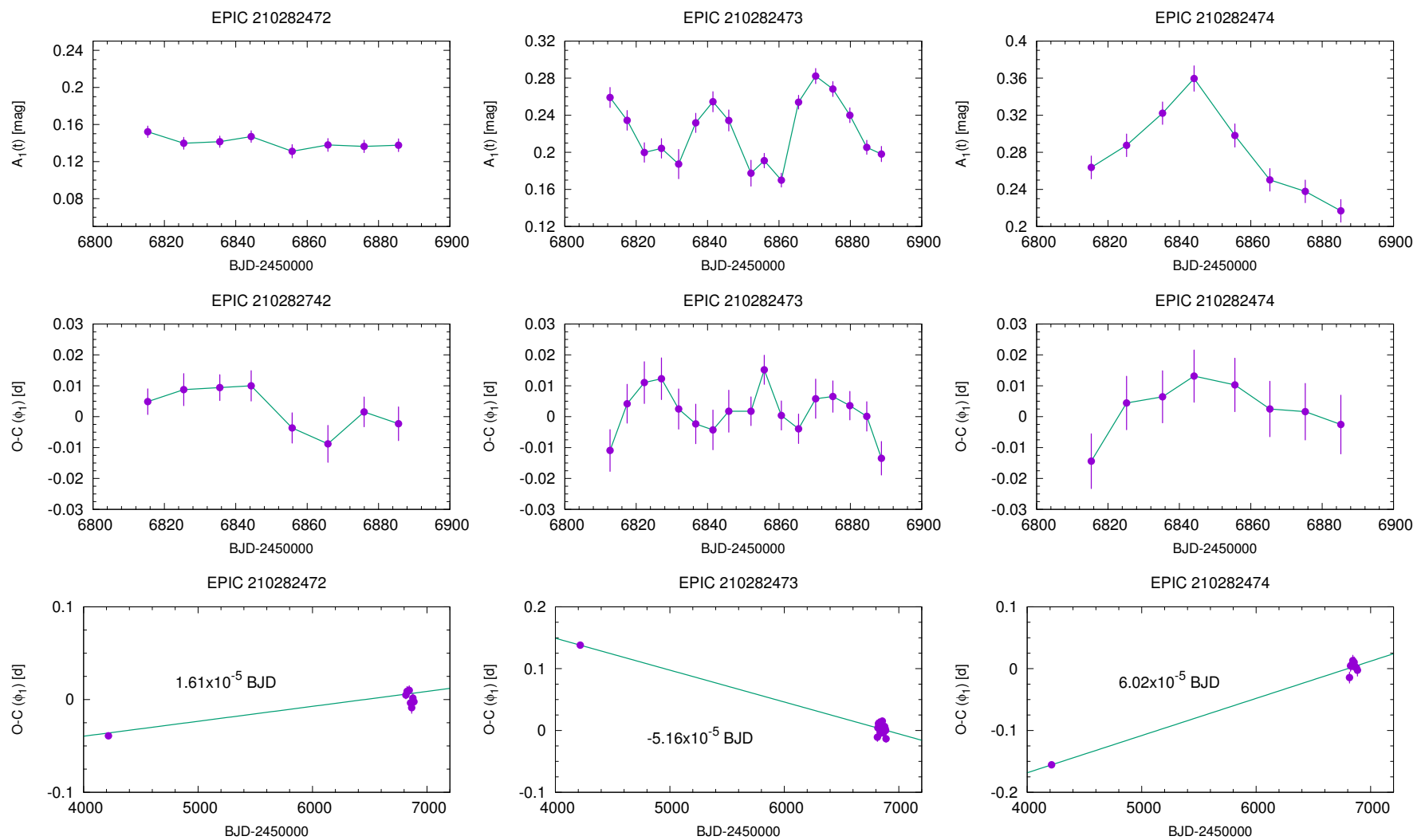

FIg. 6.- Top row: variation $A_{1}$, the amplitude of the $f_{1}$ frequency component in all three stars. Middle row: O-C variation, calculated as the variation of the $\phi_{1}$ Fourier-parameter. Bottom row: long-term period drifts of all three stars between 2007 (Moretti et al. 2009) and 2014 , the K2 observations. 
TABLE 4

PULSATION PERIODS, MODULATION PERIODS AND PHOTOMETRIC [FE/H] INDICES OF THE RR LYR TARGETS.

\begin{tabular}{lccc}
\hline \hline Object & Pulsation per. (d) & Mod. per. (d) & {$[\mathrm{Fe} / \mathrm{H}]$} \\
\hline 472 & $0.709862 \pm 5.5 \cdot 10^{-5}$ & - & $-2.16 \pm 0.10$ \\
473 & $0.619022 \pm 3.1 \cdot 10^{-5}$ & $29.8 \pm 0.9$ & $-2.64 \pm 0.10$ \\
474 & $0.630588 \pm 4.4 \cdot 10^{-5}$ & $>80$ & $-2.62 \pm 0.15$ \\
\hline
\end{tabular}

sation period of EPIC 210282473 got shorter during the $7.1 \mathrm{yr}$ separating the two data sets while the periods of EPIC 210282472 and 210282474 got longer. Other observations between the two epoch were too sparse to determine the pulsation phase and $\mathrm{O}-\mathrm{C}$ values. Therefore actual rates of period change cannot be determined yet.

\subsection{Photometric metallicities}

A great advantage of RR Lyrae stars is that we can estimate their $[\mathrm{Fe} / \mathrm{H}]$ indices from photometry alone $(\mathrm{Ju}-$ rcsik \& Kovács 1996). Nemec et al. (2013) have calibrated the relation between the light curve parameters and the spectroscopically determined metallicity values for the Kepler passband and showed that the photometric and spectroscopic $[\mathrm{Fe} / \mathrm{H}]$ indices agree to \pm 0.1 dex for all but the extremely modulated stars. We calculated the photometric $[\mathrm{Fe} / \mathrm{H}]$ values for all three stars with this method: the results are summarized in Table 4 . The accuracy intrinsic to Fourier parameter determination is \pm 0.03 dex. However, according to Nemec et al. (2013), the uncertainties of the relation are dominated by the calibration of the equation and the determination of the spectroscopic values, so we assumed higher uncertainties for all stars (Table 4 .

Of the three stars, only EPIC 210282472 has a spectroscopically determined index, $[\mathrm{Fe} / \mathrm{H}]=-2.03$ (Kirby et al. 2008) that is higher, but within the respective error bars compared to our photometric index of $-2.16 \pm$ 0.10 , assuming the same uncertainties. Moretti et al. (2009) determined the photometric metallicity for EPIC 210282473 , but their result, $[\mathrm{Fe} / \mathrm{H}]=-2.11$ is significantly higher than the K2-derived value $(-2.64 \pm 0.10)$. This discrepancy can largely be attributed to the fact that the linear relation of Jurcsik \& Kovács (1996), used by Moretti et al. (2009), overestimates the $[\mathrm{Fe} / \mathrm{H}]$ index by as much as $\sim 0.3$ dex for very metal-poor stars. The K2 data also revealed that the star is modulated, and the poor modulation phase coverage may have affected the original metallicity determination. Since the Blazhko effect in the star is well covered by the K2 data and does not show extreme amplitude changes or cycle-to-cycle variability, we consider the photometric metallicity accurate to 0.10 dex (Nemec et al. 2013).

We calculated the metallicity of the third star, EPIC 210282474 , to be $[\mathrm{Fe} / \mathrm{H}]=-2.62 \pm 0.15$. We note that if the star is indeed modulated, the partial phase coverage of the Blazhko effect could affect the photometric metallicity value. We increased the uncertainty to account for this additional factor. Overall, the three metallicity values are in good agreement with the average metallicity values of Leo IV (around -2.3 or -2.6 ) and confirm its very metal-poor nature.

\section{CONCLUSIONS}

We detected the variations of all three extragalactic RR Lyrae stars in the K2 mission Campaign 1 data. The stars are members of the ultra-faint dwarf spheroidal galaxy Leo IV (Moretti et al. 2009). These are the faintest pulsating stars measured with the Kepler space telescope so far, at a brightness level of $K p \approx 21.5$ mag.

One of the variables, EPIC 210282473, displays clear amplitude variations with a period of $29.8 \pm 0.9$ days, while EPIC 210282474 is likely modulated with a period longer than the campaign length $(80 \mathrm{~d})$. In the case of the former star, almost three modulation cycles are covered, during which the strength of the Blazhko effect and the relation between the amplitude and phase modulation both changed. These observations are the farthest measurements of the detailed parameters of the Blazkho effect at a distance of $154 \mathrm{kpc}$, and the first one beyond the Milky Way and the Magellanic Clouds. We determined the photometric $[\mathrm{Fe} / \mathrm{H}]$ indices of the stars that range between -2.16 and -2.64 , in agreement with the spectroscopic values measured in Leo IV.

We also demonstrated that with fine-tuned algorithms based on image subtraction, nearly the same faintness level can be achieved for stationary targets that is attainable for moving objects, such as TNOs. We found that unlike brighter sources, very faint but high amplitude variations do not significantly contain frequencies caused by the attitude correction maneuvers of Kepler. The implications of this exercise are far-reaching, as photometric space missions will continue to stare at crowded fields in the future. Globular clusters have already been measured during the K2 mission, and Kepler may observe Cepheid stars in another galaxy, IC 1613, in Campaign 8. The TESS and PLATO missions will employ even larger pixels that Kepler did, leading to strong crowding in several fields where image subtraction will be a necessary requirement (Ricker et al. 2014, Rauer et al. 2014). Just one, important example is the Large Magellanic Cloud within the southern continuous observing zone of TESS. The LMC contains several bright, largeamplitude variables (Cepheids for example) that will be accessible for that mission, but their photometry will present challenges very similar to the K2 observations presented here in terms of crowding and signal-to-noise ratios.

We are grateful to the referee for the constructive comments that helped to improve the paper. We thank the hospitality of the Veszprém Regional Centre of the Hungarian Academy of Sciences (MTA VEAB) where part of our work was carried out. Funding for the Kepler and K2 missions is provided by the NASA Science Mission directorate. The authors gratefully acknowledge the Kepler team, the Guest Observer Office, and Ball Aerospace, whose outstanding efforts have made these results possible. This project has been supported by the Lendület-2009, LP2012-31 and LP2014-17 Programs of the Hungarian Academy of Sciences and the OTKA grants K-104607, K-109276, and K-115709. The research leading to these results has received funding from the ESA PECS Contract No. 4000110889/14/NL/NDe, and from the European Community's Seventh Framework Programme (FP7/2007-2013) under grant agreements 
no. 269194 (IRSES/ASK) and no. 312844 (SPACEINN). L.M. was supported by the János Bolyai Research Scholarship of the Hungarian Academy of Sciences. VR acknowledges partial support by the project PRIN-MIUR (2010LY5N2T) "Chemical and dynamical evolution of the Milky Way and Local Group galaxies" (P.I.: F. Matteucci). All of the data presented in this paper were obtained from the Mikulski Archive for Space Telescopes (MAST). STScI is operated by the Association of Universities for Research in Astronomy, Inc., under NASA contract NAS5-26555. Support for MAST for non-HST data is provided by the NASA Office of Space Science via grant NNX09AF08G and by other grants and contracts.

\section{REFERENCES}

Alcock, C., Allsman, R. A., Axelrod, T. S. et al. 1996, AJ, 111, 1146

Belokurov, V., Zucker, D. B., Evans, N. W., et al. 2007, ApJ, 654, 897

Benkő, J. M., Plachy, E., Szabó, R., Molnár, L., Kolláth, Z. 2014, ApJS, 213, 31

Borucki, W. J., Koch, D., Basri, G., et al. 2010, Science, 327, 977

Brown, T. M., Ferguson, H. C., Smith, et al. 2004, AJ, 127, 2738

Da Costa, G. S., Rejkuba, M., Jerjen, H., Grebel, E. K., 2010, ApJ, 708, L121

Howell, S. B., Sobeck, C., Haas, M., et al. 2014, PASP, 126, 398

Jurcsik, H., Kovács, G. 1996, A\&A, 312, 111

Jurcsik, J., Sdor, Á.; Szeidl, B., et al., 2009, MNRAS,400, 1006

Kirby, E. N., Simon, J. D., Geha, M., Guhathakurta, P., Frebel, A. 2008, ÄpJ, 685, 43

Lenz P., Breger M. 2005, CoAst, 146, 53

Molnár, L., Szabó, R., Moskalik, P. A., et al., 2015, MNRAS, 452, 4283

Monet, D. G., Levine, S. E.; Canzian, B., et al. 2003, AJ, 125, 984

Moretti, M. I., Dall'Ora, M., Ripepi, V., et al. 2009, ApJ, 699, 125

Nemec, J. M.; Cohen, J. G.; Ripepi, V., et al. 2013, ApJ, 773, 181

Olling, R. P., Mushotzky, R., Shaja, E. R., et al., 2015, Nature, 521, 332

Pál, A. 2009. PhD thesis. Eötvös Loránd University, Budapest, Hungary (arXiv:0906.3486)

Pál, A. 2012, NINRÁs, 421,1825

Pál, A., Szabó, R., Szabó, Gy. M., et al., 2015, ApJ, 804 L45

Rauer, H., Catala, C., Aerts, C., et al., 2014, Exp. Astr., 38, 249

Ricker, G. R. Winn, J. N., Vanderspeck, R., et al, 2014, Proc. SPIE, 9143, 914320

Sand, D. J., Seth, A., Olszewski, E. W., et al., 2010, ApJ, 718, 530

Simon, J. D., Geha, M. 2007, ApJ, 670, 313

Stetson, P. B., Fiorentino, G., Bono, G., et al., 2014, PASP, 126, 616

Szabó, R., Kolláth, Z., Molnár, L., et al. 2010, MNRAS, 409, 1244

Szabó, R., Sárneczky, K., Szabó, Gy. M., et al. 2015, AJ, 149, 112

Soszynski, I., Udalski, A., Szymanski, M., et al. 2009, AcA, 59, 1

Soszynski, I., Udalski, A., Szymanski, M. K., et al., 2010, AcA 60,165

Soszynski, I., Udalski, A., Szymanski, M. et al., 2014, AcA, 64, 177

Szczygieł, D. M.; Fabrycky, D. C. 2007, MNRAS, 377, 1263 